\title{
Bone Density in Thalassemic Children during the Course of the Disease
}

\author{
S. DOXIADIS, ${ }^{(25)}$ E. GEORGAKI, D. PAPAMICHAEL, S. PAPADAKOU-LAGOGIANNI, AND \\ P. LAPATSANIS \\ Institute of Child Health and Agriculture College of Athens, Athens, Greece
}

\begin{abstract}
Summary
The study was carried out on $\mathbf{5 0}$ thalassemic children and 20 control subjects aged 5-15 years. The cortical thickness in thalassemic children was less than in control subjects. The bone loss was more obvious in girls $(P<0.0005)$ than in boys $(P<0.05)$. The greater bone loss in girls could not be explained by the level of blood hemoglobin because in both sexes the mean value was the same $(7.3 \pm 0.5 \mathrm{~g} / \mathrm{dl})$.

Of the 12 thalassemic children observed for 9-24 months, 9 showed no significant bone change, 2 showed an improvement of cortical thickness and only 1 showed bone loss. Of the 11 thalassemic children observed for $25-48$ months, 4 showed significant bone loss and 7 no change of the bone density. In both groups the mean value of pretransfusion blood $\mathrm{Hb}$ was $7.3 \mathrm{~g} / \mathrm{dl}$ and the sex distribution was equal. A relationship was found in both control and thalassemic children between bone density and urine phosphorus to urine creatinine ratio.
\end{abstract}

\section{Speculation}

The bone density is less in thalassemic than in control children and this difference is more obvious in girls. An investigation of the hormonal factors which are related to the bone density might give useful information concerning the hormonal status during the prepubertal period in thalassemic girls. This knowledge may help in preventing the severe bone loss in girls. The findings of the investigation may have more practical application in thalassemic girls, who do not need blood transfusions because they have blood hemoglobin values of 7-10 $\mathrm{g} / \mathrm{dl}$, but who have the bone lesions of thalassemia. In each individual patient the bone loss requires more than 24 months to become radiologically obvious when the pretransfusion blood hemoglobin is $7-8 \mathrm{~g} / \mathrm{dl}$. The bone loss during the course of the disease could be reduced with higher pretransfusion blood hemoglobin values with the complication of iron overload. From a previous study it was shown that thalassemic children have abnormally high phosphaturia. It is therefore likely that phosphorus administration could have beneficial effect on the bone density. Further investigations should be performed to assess the beneficial effect of different pretransfusion blood hemoglobin values and/or the extra oral phosphorus administration on the bone density in thalassemic children. Apart from the metabolism of phosphorus, that of calcium should also be investigated in thalassemic patients. It will be advisable to measure both ratios (UP:UCr and UCa:UCr) and to relate them to bone density. If a relationship is found then these two ratios can replace the radiologic examination.

The bone density in thalassemic children has been studied very little $(13,15)$ and in none of the studies have the changes during the course of the disease been investigated. The present work describes 1) a study of the bone density in thalassemic children and the changes of the density during the course of the disease in each thalassemic patient and 2) an attempt to develop a screening test for the condition of bone density in thalassemic patients.

\section{METHODS}

In all children duplicate measurements of cortical thickness on $x$-ray films of the metacarpals of both hands were made (9). The measurement was made in the thalassemic children every 6 months and in the control subjects once. The Exton-Smith index (6) was used for the estimation of the bone density of each metacarpal because in it the length of metacarpal bone is taken into consideration and the length of the second left metacarpal bone is indirectly related to 1) the weight of the skeleton (bone density) and 2) the height of the body. This is important since thalassemic children are retarded in height in comparison to normal children. The method of Horsman et al. (9) was also used 1) for more accurate results and 2) to follow individual changes of bone density (24).

Control and thalassemic children did not receive any food for at least $12 \mathrm{hr}$ before the biochemical investigations (overnight). They emptied their bladders in the morning; a collection of urine was made $3 \mathrm{hr}$ later and at the same time a sample of blood was taken. Urine phosphorus $(P)$ to urine creatinine $(C r)$ concentration ratio (UP:UCr) was measured. Urinary $P$ and urinary $C r$ were measured as reported in a previous paper (5).

\section{MATERIALS}

The group under investigation comprised the following. 1) Fifty children ( 22 boys and 28 girls) with an equal age distribution (5-15 years) who received regular blood transfusions in the day clinic for thalassemia of the Aghia Sophia Children's Hospital. All had hepatosplenomegaly, anemia, normoblastosis, an abnormal amount of fetal hemoglobin, and typical thalassemic lesions of skull and other bones. In each case both parents were heterozygous for thalassemia as shown by erythrocyte morphology and hemoglobin electrophoresis. Of these patients 12 were observed for 9-24 months and 11 for 25-48 months. 2) Twenty control subjects ( 12 boys and 8 girls) aged 5-15 years who were admitted to the hospital for adenoidectomy or tonsillectomy. All control subjects had blood hemoglobin over $11.5 \mathrm{~g} / \mathrm{dl}$.

\section{RESULTS}

Figure I shows the mean value and the SD for age and bone density in the thalassemic and control boys. Thalassemic boys had smaller bone density than controls with an equal age distribution $(P<0.05)$. If we disregard the two thalassemic cases with very low values of bone density, the difference in the bone density had a statistical significance at the level of $10 \%(0.1>P>0.05)$.

Figure 2 shows the mean values and the SD for age and bone density in the thalassemic and control girls. In the thalassemic girls the bone density was markedly less than in the control subjects $(P<0.0005)$ with an equal age distribution. 


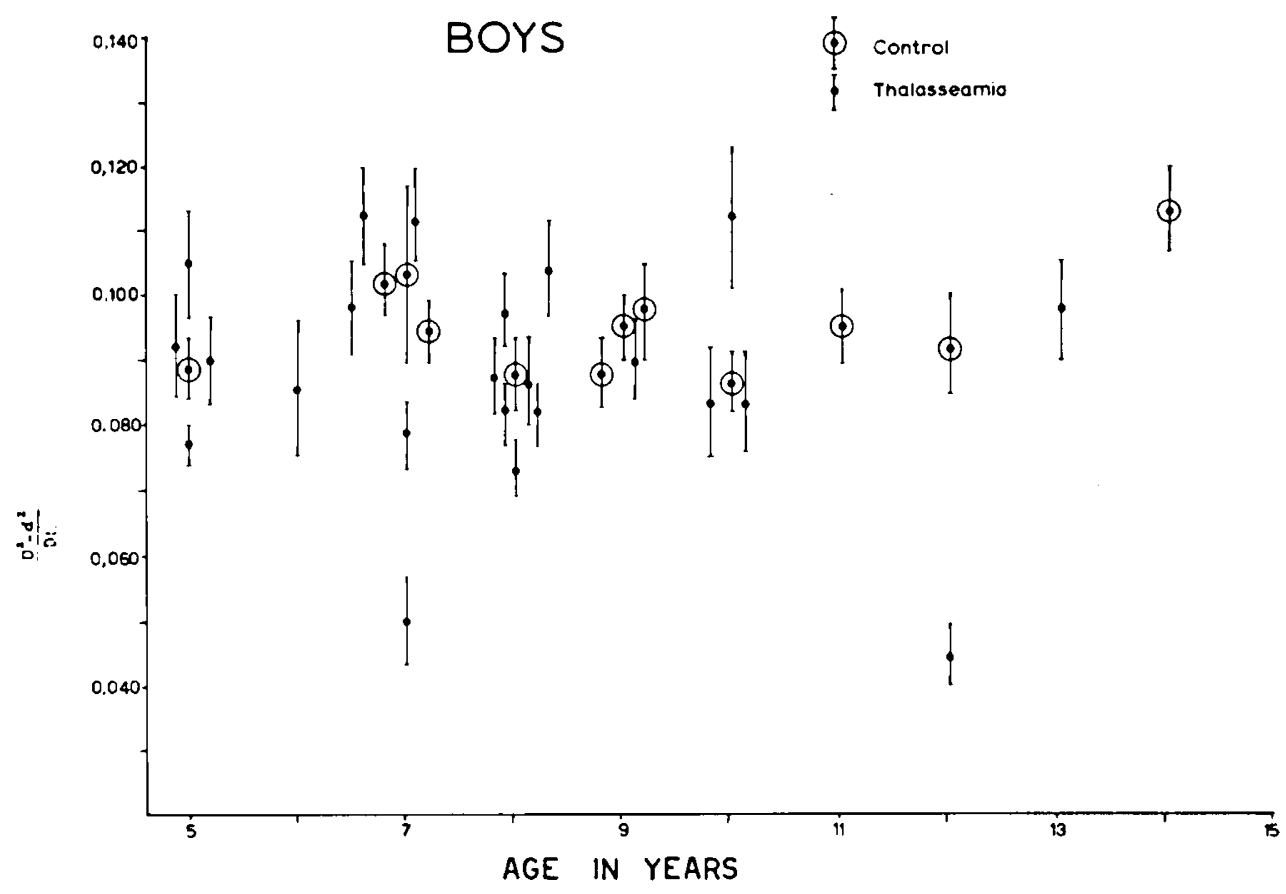

Fig. 1. Bone density in the thalassemic and control boys. $\frac{D^{2}-d^{2}}{D L}, \vec{x}$ is the average bone density in each boy evaluated from the 20 measurements. I: $\frac{\mathrm{D}^{2}-\mathrm{d}^{2}}{\mathrm{DL}}, \mathrm{x}$ is the bone density in each boy with $95 \%$ confidence interval according to the formula $\mathrm{x}=\overline{\mathrm{x}} \pm \frac{2.093}{\sqrt{20}}$ s, as in the chapter on methods.

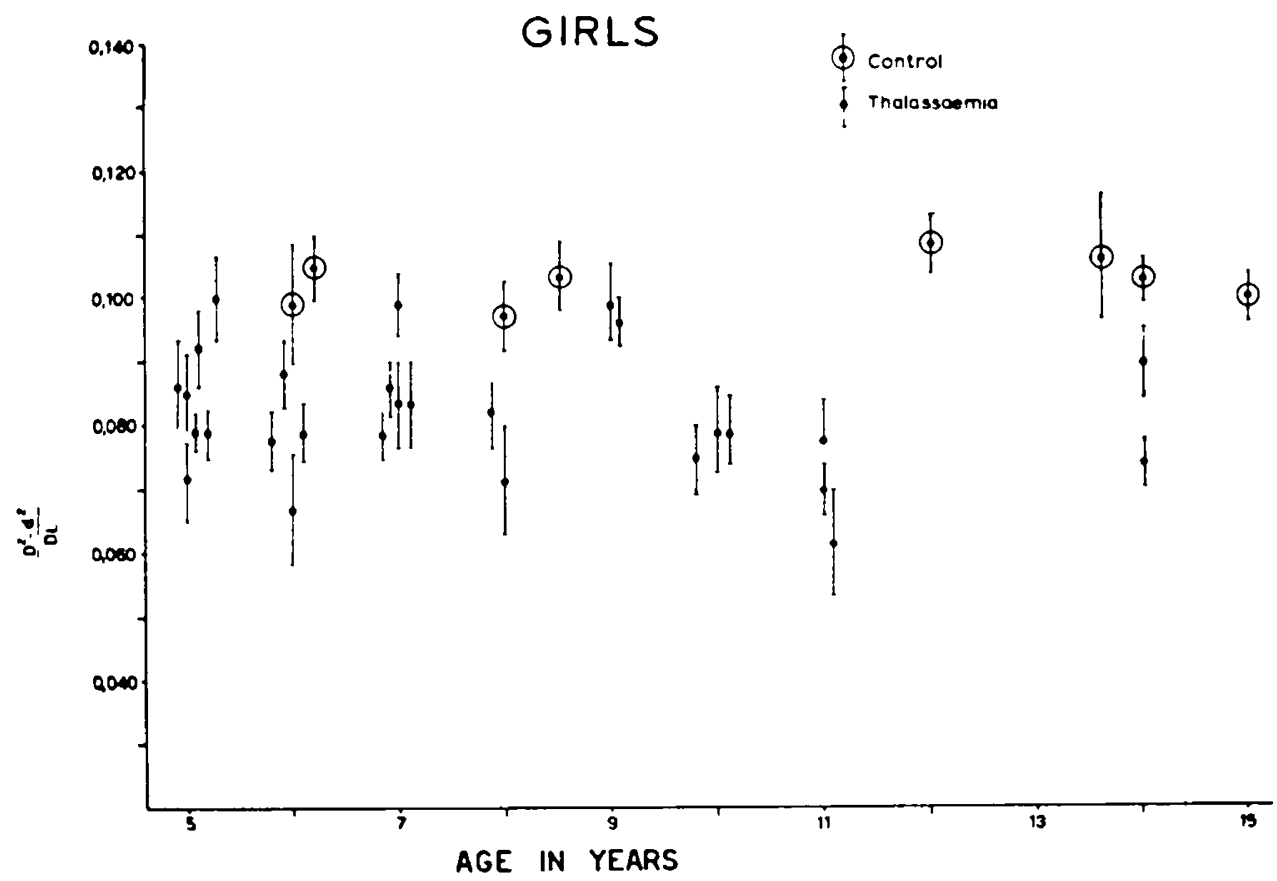

Fig. 2. Bone density in the thalassemic and control girls (explanation of symbols as in Figure 1).

Table 1 shows the mean values and the SD for age, blood $\mathrm{Hb}$, and bone density in all thalassemic children. As it may be seen the bone density was less in the girls than in the boys but the difference was not statistically significant $(0.10>P>0.05)$.

Figure 3 shows the bone density changes in 12 thalassemic children for a period of observation of 9-24 months. Of the 12 children, 9 showed no significant change, 2 showed an improvement of cortical thickness, and only 1 showed bone loss.

Figure 4 shows the bone density changes in 11 thalassemic children for a period of observation of 25-48 months. Of the 11 children, 4 showed a significant bone loss and 7 showed no change.

Figure 5 shows the relation between UP:UCr ratio and bone density in both groups of children. A negative correlation between the two measurements was found $(P=0.05)$. If we disregard the one thalassemic case with a very low value of bone density and normal UP:UCr ratio (Fig. 5), the correlation between the two parameters was statistically significant $(r-0.5052 ; P<0.001)$. As the UP:UCr ratio increased the bone density decreased. 


\section{DISCUSSION}

The estimation of bone density by simple observation of radiograms is not reliable $(2,7,18)$, and other more accurate methods have been used in the last 15 years. Morphometric methods were used by some authors $(1,6,16)$ and scanning techniques by others $(10,16,20)$. Although the complicated scanning techniques are considered as more accurate, the morphometric methods do not fall far behind and there is a close relationship between these two methods $(12,16)$. According to Sorenson and Cameron (20), the presence of fat in the medullary cavity of bone leads to serious error with scanning techniques. Cortical bone is frequently replaced by fat in bone disease. These authors (20) suggest that a reasonably accurate correction for effects of fat in the marrow cavity could be made from radiographs and appropriate correction curves (21). Thalassemic children have cortical thinning and enlarged marrow cavity with a decrease of erythropoietic tissue in

Table 1. Bone density in thalassemic children ${ }^{1}$

\begin{tabular}{lcccc}
\hline & No. of cases & $\begin{array}{c}\text { Bone density } \\
\left(\frac{\mathrm{D}^{2}-\mathrm{d}^{2}}{\mathrm{DL}}\right)\end{array}$ & $\begin{array}{c}\text { Blood Hb, } \\
(\mathrm{g} / \mathrm{dl})\end{array}$ & $\begin{array}{c}\text { Age } \\
(\mathrm{yrs})\end{array}$ \\
\hline Boys & 22 & $0.087 \pm 0.016$ & $7.3 \pm 0.5$ & $8 \pm 2.1$ \\
Girls & 28 & $0.081 \pm 0.009$ & $7.2 \pm 0.9$ & $8 \pm 2.7$ \\
\hline
\end{tabular}

' $t=1.472 ; 0.10>P>0.05$. $t:$ difference in bone density between thalassemic boys and girls; $\mathrm{n}_{1}$ : number of thalassemic boys $(n=22) ; \bar{x}_{1}$ : mean value of bone density $\left(\frac{D^{2}-d^{2}}{D L}\right)$ in the thalassemic boys; $s_{1}$ : standard deviation of the measurements in the thalassemic boys; $\mathrm{n}_{2}$ : number of thalassemic girls $(n=28)$; $\bar{x}_{2}$ : mean value of the bone density $\left(\frac{D^{2}-d^{2}}{D L}\right)$ in the thalassemic girls; $s_{2}:$ standard deviation of measurements in the thalassemic girls; $t=\sqrt{\frac{n_{1} \cdot n_{2}}{n_{1}+n_{2}}} \cdot \frac{\bar{x}_{1}-\bar{x}_{2}}{s}$, where $s=$ $\sqrt{\frac{n_{1} s^{2}+n_{2} s_{2}{ }^{2}}{n_{1}+n_{2}-2}}$ as reported in Snedecor (19) in the chapter on methods. bone marrow due to transfusions. The peculiar bone lesions in thalassemic children and the findings of Sorenson and Cameron (20) led us to use radiograms for the estimation of the bone density in thalassemic children. Johnston and Roseman (13) reported less cortical thickness in thalassemic children than in control subjects, but Liakakos et al. (15) found no difference. Both groups of investigators, however, used a single measurement of only one metacarpal. Our own findings based on two measurements of all 10 metacarpals showed a significant difference and confirmed the findings of Johnston et al. (18). The less dense bone of thalassemic children can explain the greater tendency of these children to fractures $(4,17)$. These would have been more frequent if the thalassemic children had not had a lower level of physical activity. To increase the bone density one would have to keep hemoglobin values at a higher level (23) with the concomitant advantages, but, also disadvantages and difficulties of such a regime. Another way would be to increase the intake of vitamin $D$, phosphorus or calcium. Theoretically, the risk of calcification of soft tissues and kidneys by the increased administration of phosphorus and/or calcium and/or vitamin D could not be excluded but this risk is very low, almost nonexistent. Our own experience with phosphorus administration (to be published) shows that this has a beneficial effect on bone density in thalassemic children and none of these patients showed an increase blood calcium and/or urinary calcium to urinary creatinine ratio and/or nephrocalcinosis.

In the thalassemic children, however, the bone density of the girls was less than that of the boys, but the difference had a statistical significance at the level of $10 \%$. The level of blood hemoglobin alone could not explain the sex difference of bone density in thalassemic children. Other factors (hormones, etc.) must be responsible for this. This finding needs further investigation because it may be of importance, especially for the thalassemic females with intermediate type of the disease, who do not need transfusion, reach adult life, and are therefore more likely to have osteoporosis and to sustain fractures.

The course of bone changes in individual patients has been studied in women with osteoporosis $(9,10)$, but not in thalassemic children. In normal children an increase of bone density takes

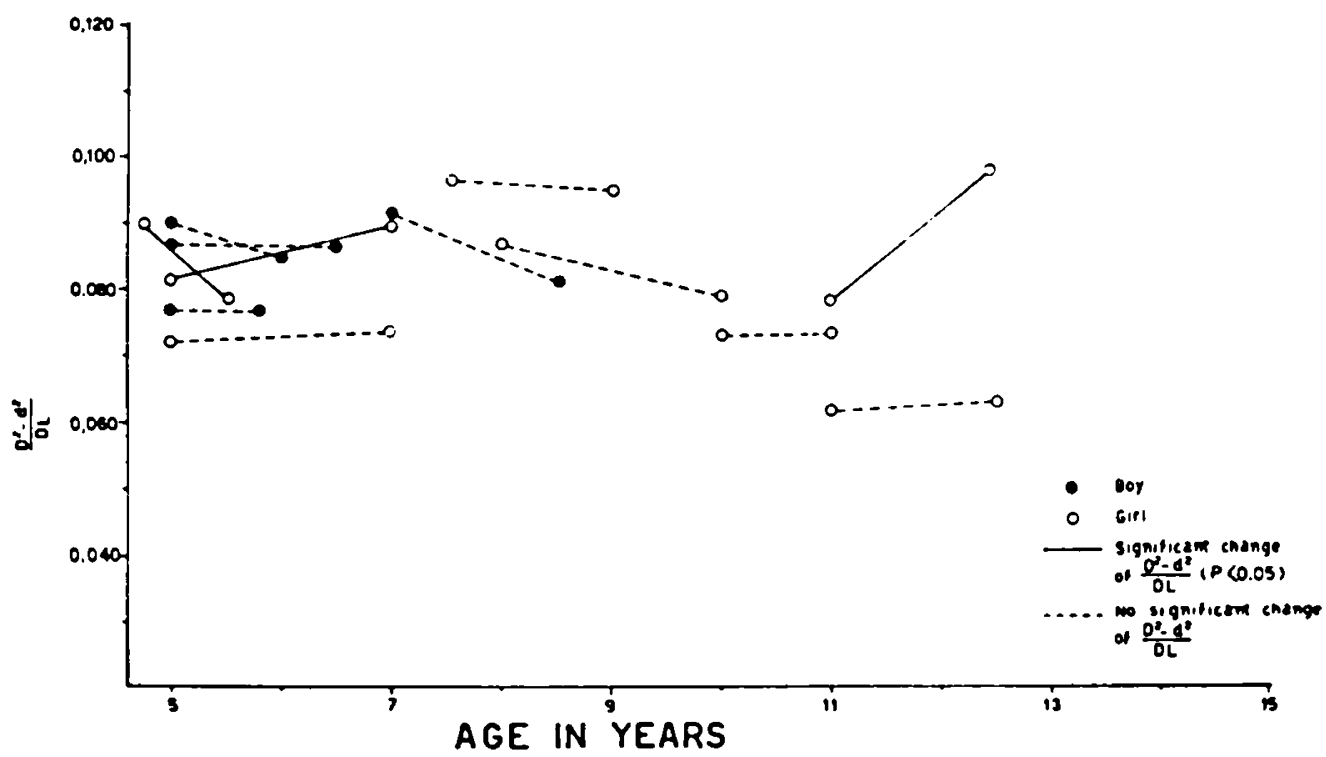

Fig. 3. Individual bone density changes in thalassemic children (period of observation 9-24 months). $1=\sqrt{\frac{n_{1} n_{2}}{n_{1}+n_{2}}} \cdot \frac{\bar{x}_{1}-\bar{x}_{2}}{s}$, where $s=$ $\sqrt{\frac{n_{1} s_{1}{ }^{2}+n_{2} s_{2}{ }^{2}}{n_{1}+n_{2}-2}} ; n_{1}: 20$, the number of observations of the 10 metacarpal bones, twice; $\bar{x}_{1}:$ the mean value of bone density $\left(\frac{D^{2}-d^{2}}{D L}\right)$ in the 10 metacarpal bones, twice, from the first $x$-ray film; $s_{1}:$ SD of the 20 measurements of the bone density from the first $x-r a y$ film. $n_{2}: 20$, the number of observations of the 10 metacarpal bones, twice; $\bar{x}_{2}:$ mean value of the bone density $\left(\frac{D^{2}-d^{2}}{D L}\right)$ in the 10 metacarpal bones, twice from the second film of the same thalassemic child, 9-24 months after the first $x$-ray film; s: SD of the 20 measurements of the bone density from the second $x-r a y ~ f i l m: t$ expresses the changes of bone density in the same individual with an interval of 9-24 months between the two x-ray films. 


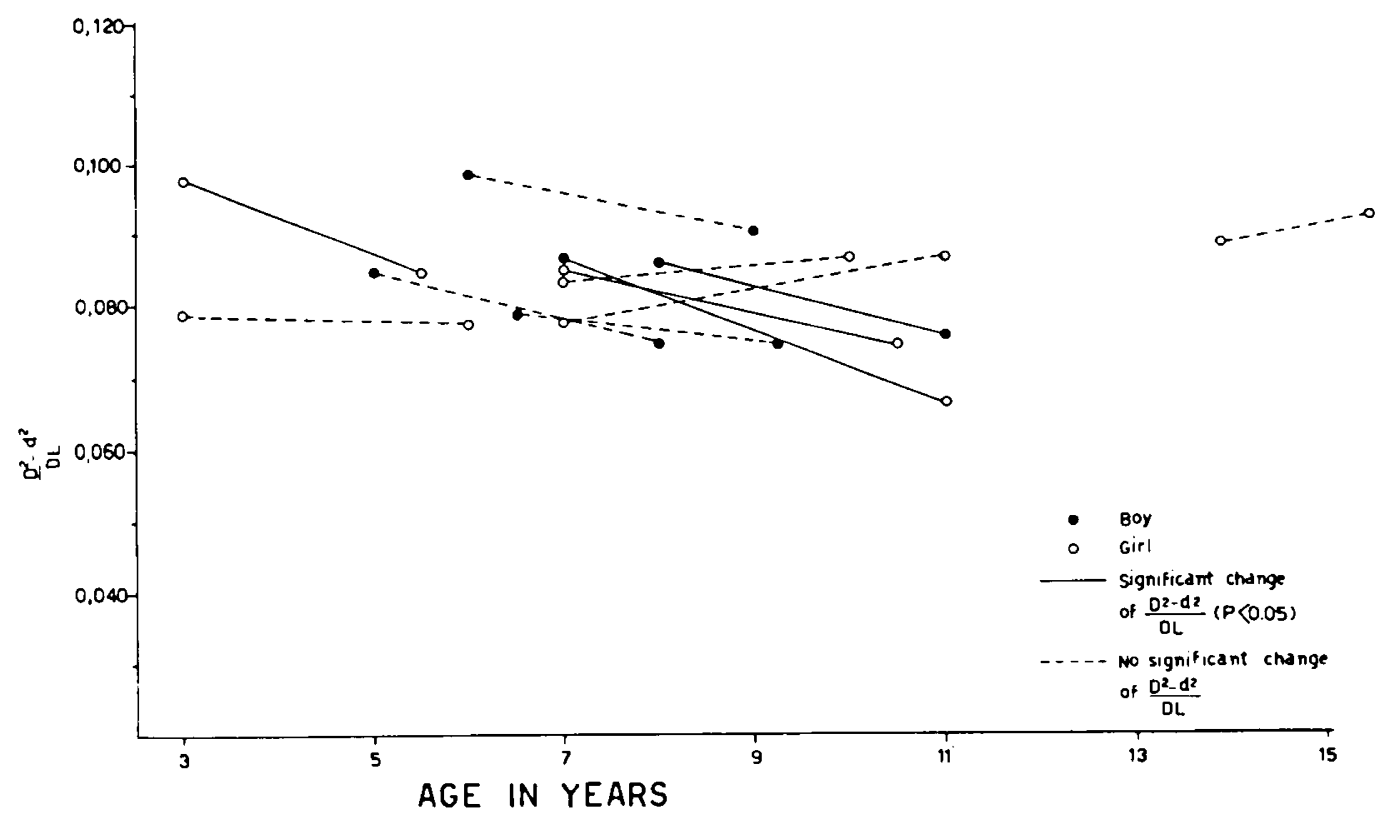

Fig. 4. Individual bone density changes in thalassemic children. Period of observation $25-48$ months. (Explanation of symbols as in Figure 3.)

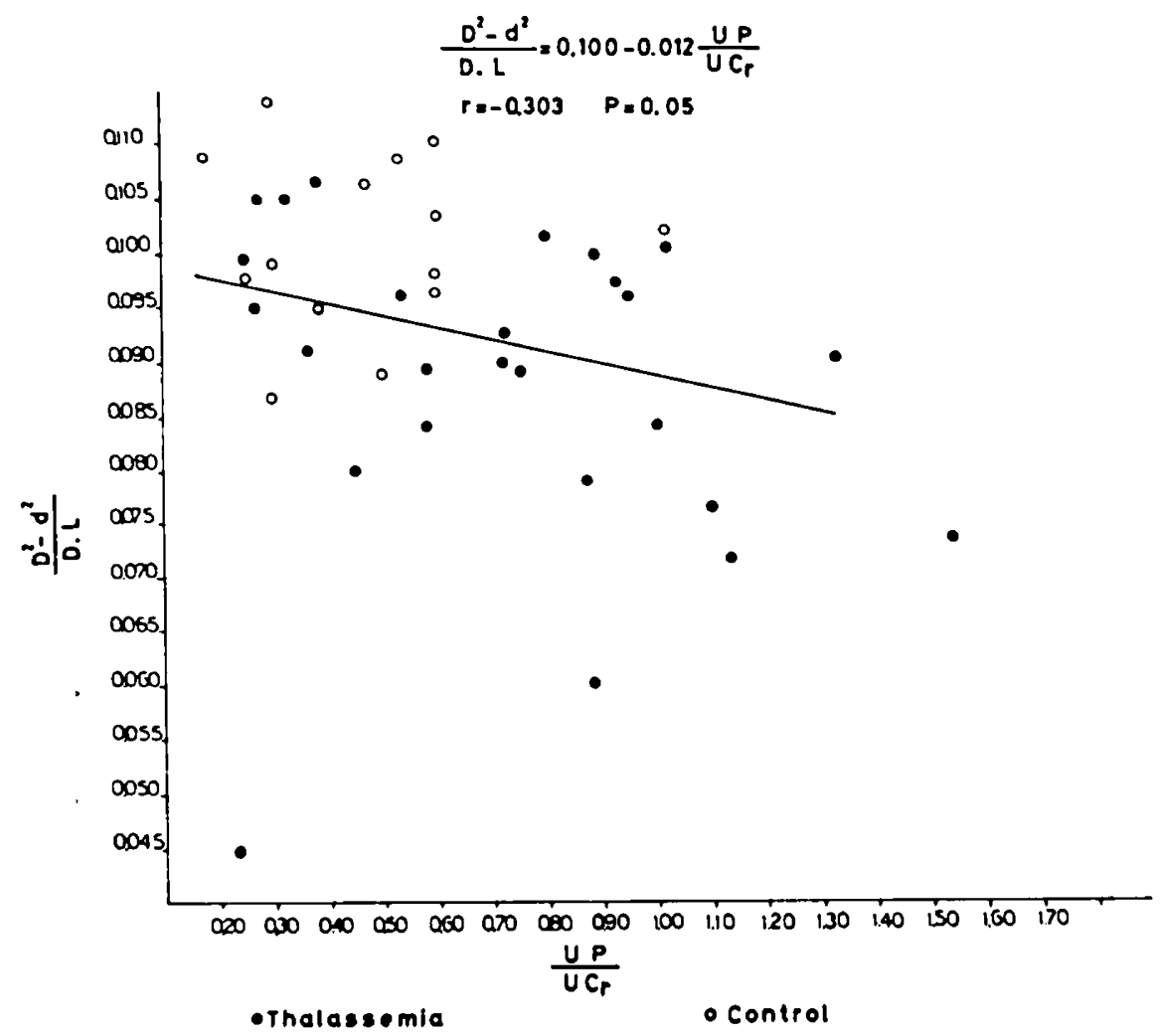

Fig. 5. Bone density and UP:UCr ratio in the thalassemic and control children $\frac{D^{2}-d^{2}}{D L}$ : average bone density in each child evaluated from 20 measurements. UP:UCr: urine phosphorus $(\mathrm{P})$ to urine creatinine $(\mathrm{Cr})$ concentration ratio.

place during growth (8). In thalassemic patients there was either no change or a decrease of bone density. These developments, however, required more than 24 months of radiologic observation, when the pretransfusion blood $\mathrm{Hb}$ was $7-8 \mathrm{~g} / \mathrm{dl}$.

By using the present technique for estimation of individual changes of bone density in a thalassemic child under a vigorous or low transfusion program we could obtain information concerning the effect of age, of growth spurt, and of puberty on the bone density.
Bone is one of the tissues mainly affected in thalassemic children. A knowledge of the condition of the bones is therefore essential for the assessment of the whole state of health. In order to avoid undue exposure to $\mathrm{x}$-rays we should attempt to find other indirect indications of the condition of bones. Hurwitz et al. (11) found in rachitic rats and Lapatsanis et al. (14) in rachitic children a close inverse relationship between serum phosphorus and rachitic bone lesions but not between serum calcium and the severity of bone lesions. The children in our study did not receive any 
food for $12 \mathrm{hr}$ and we can assume that the urinary $P$ was coming from the bones $(3,22)$. Of the 11 thalassemic children with a UP:UCr ratio less than 0.6 only 3 had abnormally low bone density. In 15 thalassemic children with UP:UCr ratio 0.6 or higher the bone density was at low normal or abnormally low limits. If we disregard the case with very low bone density but with normal UP:UCr ratio, then there was a strong negative correlation between UP:UCr ratio and bone density. If this finding is confirmed by further observations, the measurement of this UP:UCr ratio could reduce the number of radiologic examinations in thalassemic patients.

\section{CONCLUSION}

Fifty thalassemic children ( 22 boys and 28 girls) and 20 control subjects were studied. Twelve of the thalassemic patients were observed for 9-14 months and 11 for $26-48$ months. The bone density in thalassemic children was $0.087 \pm 0.016$ in boys and $0.081 \pm 0.009$ in girls. The bone density is less in thalassemic than in control children. In thalassemic children the bone density is smaller in girls than in boys. The individual bone loss of thalassemic children in this age group (5-15 years) needs more than 24 months to become radiologically obvious when the pretransfusion blood hemoglobin is $7-8 \mathrm{~g} / \mathrm{dl}$. A relationship was found between the ratio of urine phosphorus to urine creatinine concentration and the bone density $(P=0.05)$.

\section{REFERENCES AND NOTES}

I. Barnett, E., and Nordin, B. E. C.: The radiological diagnosis of osteoporosis, a new approach. Clin. Radiol., 11: 166 (1960).

2. Bland, J. H., Soule, A. B., VonBuskirk, F. W., Brown, E., and Clayton, R. V.: A study of inter- and intra-observer error in reading plain roentgenograms of the hands "To err is human." Amer. J. Roentgenol., 105: 853 (1969).

3. Bijvoet, O. L. M.: Renal phosphate excretion in man. Folia Med. Neerl., 15: 84 (1972).

4. Caffey, J.: Cooley's Anemia: A review of the roentgenographic findings in the skeleton. Amer. J. Roentgenol. Rad. Ther. Nucl. Med., $78: 381$ (1957).

5. Doxiadis, S.. Angelis, C., Karatzas. P., Vretos, C., and Lapatsanis, P.: Genetic aspects of nutritional rickets. Arch. Dis. Childhood 51: 83 (1976).

6. Exton-Smith, A., Millard. P., Payne, P., and Wheeler, E.: Method for measuring quantity of bone. Lancet, ii: 1153 (1969).

7. Feist, J. H.: The biologic basis of radiologic findings in bone disease. Radiol. Clin. N. Amer., 8: 183 (1970).
8. Gryfe, C., Exton-Smith, A., Payne, P., and Wheeler, E.: Pattern of development of bone in childhood and adolescence. Lancet, $i: 523$ (1971).

9. Horsman, A., Nordin, B. E. C., and Gallagher, J. C.: Sequential bone changes in treated and untreated postmenopausal women. Israel J. Med. Sci., 10: 1471 (1974).

10. Horsman, A., Nordin, B. E. C., Gallagher, J. C.. Kirby, A. P., Milner. M. R., and Simpson, M.: Observation of sequential changes in bone mass in postmenopausal women: a controlled trial of oestrogen and calcium therapies. Calcif. Tissue Res. Suppl., 22: 217 (1977).

II. Hurwitz, S., Stacey, R. E., and Bronner, F.: Role of vitamin D in plasma calcium regulation. Amer. J. Physiol., 216: 254 (1969).

12. Ikkos, D., Velentzas, C.. Katsichtis, C.. Ntalles, K., and Stergiou, L.: Effect of age on bone mass of the Greek population aged 15-90 years, morphometric observations. Iatriki, 21: 287 (1971).

13. Johnston, F., and Roseman, J.: The effects of more frequent transfusions upon bone loss in thalassemia major. Pediat. Res., 1: 479 (1967).

14. Lapatsanis, P., Makaronis, G., Vretos, C., and Doxiadis, S.: Two types of nutritional rickets in infants. Amer. J. Clin. Nutr., 29: 1222 (1976).

15. Liakakos, D., Ikkos, D., Ntalles, K., Vlachos, P., Valavani, M., and Vitsarakohila, E.: Bone mass of children suffering from homozygous $\beta$-thalassemia. Mater. Med. Greca, 4: 283 (1976).

16. Mazess, R. B., Cameron, J. R., and Sorenson, J. A.: A comparison of radiological methods for determining bone mineral content. In: J. R. Cameron: Proceedings of the Bone Measurement Conference, USAEC. USA (1970).

17. Pearson. H.: Thalassemia major. In: W. Nelson, V. Vaughan, and J. McKay: Text Book of Pediatrics, p. 1058 (Saunders, Philadelphia. 1969).

18. Sherman. R. S.: The nature of radiologic diagnosis of diseases of bone. Radiol. Clin. N. Amer., 8: 227 (1970).

19. Snedecor, W. G.: Statistical Methods, pp. 48, 91 (The Iowa State University Press, Ames, Iowa, 1961).

20. Sorenson. J. A., and Cameron, J. R.: Transmission scanning techniques. In: G. J. Hine and J. A. Sorenson: Instrumentation in Nuclear Medicine, p. 349 (Academic Press, New York, 1974).

21. Sorenson, J. A., and Mazess, R. B.: Effects of fat on bone mineral measurement. In: Bone Measurement Conference, USAEIC, p. 255 (1970).

22. Walton, R. J., and Bijvoet, O. L. M.: Nomogram for derivation of renal threshold phosphate concentration. Lancet, $i i, 309$ (1975).

23. Wolman. I. J.: Transfusion therapy in Cooley's anemia: growth and health as related to long-range hemoglobin levels. A progress report. Ann. N. Y. Acad. Sci., 119: 736 (1964).

24. The bone density and the individual changes in each child were calculated as reported by Snedecor (19). Details concerning the calculations of the bone density in each child, the individual changes and the comparison of the bone density between control and thalassemic children will be sent by the authors upon request.

25. Requests for reprints should be addressed to: Dr. Spyros Doxiadis, Institute of Child Health, Aghia Sophia Children's Hospital, Athens 617 (Greece).

26. Received for publication January 13, 1977.

27. Accepted for publication October 18, 1977. 